Voix et Images

voixetimages

\title{
Une société, un récit de Micheline Cambron
}

\section{Laure Hesbois}

Volume 16, numéro 1 (46), automne 1990

Les correspondants littéraires d'Alfred DesRochers

URI : https://id.erudit.org/iderudit/200883ar

DOI : https://doi.org/10.7202/200883ar

Aller au sommaire du numéro

Éditeur(s)

Université du Québec à Montréal

ISSN

0318-9201 (imprimé)

1705-933X (numérique)

Découvrir la revue

Citer cet article

Hesbois, L. (1990). Une société, un récit de Micheline Cambron. Voix et Images, 16(1), 154-156. https://doi.org/10.7202/200883ar d'utilisation que vous pouvez consulter en ligne.

https://apropos.erudit.org/fr/usagers/politique-dutilisation/ 


\section{Une société, un récit de Micheline Cambron}

\section{par Laure Hesbois, Université laurentienne}

À première vue, il paraît surprenant de rassembler dans la même étude un disque de Beau Dommage, une série d'articles sur l'éducation, des monologues d'Yvon Deschamps, des poèmes de Miron, une pièce de Tremblay et un roman de Ducharme, et plus surprenant encore de vouloir traiter tous ces textes comme des récits. Et quand l'auteur prétend, de surcroît, tirer de tout cela une vision cohérente de la société québécoise de 1967 à 1976, l'audace confine presque à la témérité. C'est donc l'esprit critique en éveil qu'on aborde l'essai de Micheline Cambron intitulé: Une société, un récit. Discours culturel au Québec (1967-1976) ${ }^{1}$. Avant même de commencer la lecture, on se dit que l'idée est trop séduisante pour être juste et on se promet de découvrir la faille. On est à l'affút de la proposition douteuse, de l'affirmation gratuite, de la faute de raisonnement, voire de l'omission susceptible d'invalider la démonstration. Mais c'est compter sans le talent de l'auteur et à peine est-on entré dans le texte qu'on se laisse emporter par la clarté des définitions, la rigueur de l'argumentation, la pertinence des exemples et la clarté du style. Avec une clairvoyance étonnante, l'auteur semble même prévoir les objections et s'arrange pour les réfuter avant qu'on aît eu le temps de les formuler.

Pour commencer, elle propose une définition dynamique du récit, qui lui permet d'intégrer une grande variété de textes et de justifier, du même coup, l'hétérogénéité des matériaux à laquelle je faisais allusion plus haut. Cette définition s'appuie sur une analyse critique des travaux de narratologie les plus en vue (Propp, Greimas, Genette) dont elle dégage les postulats, sans craindre d'en indiquer les limites. À la suite de Ricœur, elle nous invite à considérer la narrativité moins comme un attribut particulier de certains textes que comme une activité structurante reliée à la dimension temporelle du discours (p. 28) et à voir dans le récit non plus un objet clos mais un mode inné d'organisation du sens pour l'esprit humain (p. 19) et, par conséquent, un processus actif d'organisation du discours social commun. Ce concept, inspiré de Fossaert et de Gramsci, fait à son tour l'objet d'une mise au point qui aboutit à la notion de discours culturel, à savoir ce qui dans le brouhaha de nos pratiques quotidiennes passe par la médiation de la parole (p. 34). A partir de là, le choix du corpus se justifie aisément. Il suffit de sélectionner des œuvres ratifiées par l'opinion publique, c'est-à-dire jouissant du succès populaire et/ou de la faveur des spécialistes. Et à cet égard, il 
ne fait aucun doute que, à l'exception peut-être du roman de Ducharme, plus prisé des intellectuels que du grand public, les œuvres retenues répondent à ce double critère.

Cela fait, l'auteur entreprend donc de construire le récit commun $d u$ discours culturel québécois de 1967 à 1976 . L'idée générale est qu'il s'agit d'un discours hégémonique conservateur, travaillé par des forces de rupture, dont témoignent différents procédés de mise à distance. La démonstration se fait en trois temps: 1) la construction d'un modèle, à partir de deux exemples relativement simples, à savoir les disques de Beau Dommage et les articles de Lysiane Gagnon; 2) l'application de ce modèle à deux cuvres orales, c'est-àdire les monologues de Deschamps et une pièce de Tremblay; 3) la vérification des résultats obtenus sur deux œuvres écrites, les poèmes de Miron et un roman de Ducharme.

En comparant entre eux trois disques successifs du groupe Beau Dommage, l'auteur fait ressortir un certain nombre de différences qui, selon elle, correspondent à autant de changements dans la mentalité collective. Ces différences portent moins sur les thèmes que sur la structuration du récit, à savoir la construction du sujet, la représentation du temps, la logique des actions et l'organisation axiologique des textes. On passe d'un univers clos et rassurant, style album de famille, fondé sur. la connivence et la valorisation de souvenirs communs, à un univers de conflits, où les événements se multiplient de façon inquiétante et où le seul consensus possible est celui d'une solitude partagée. Le même danger d'éclatement peut se lire, d'une autre façon, dans le drame de l'enseignement, tel qu'il est évoqué dans les articles de Lysiane Gagnon. La langue y est présentée comme un héritage menacé, un "bien" à conserver, et tout changement dans ce domaine perçu comme une dégradation. Mais cette prise de position conservatrice n'empêche pas l'auteur de dénoncer l'incurie des pouvoirs publics et s'accompagne, paradoxalement, d'une polémique en faveur d'une réforme de l'enseignement. Il y a là une contradiction flagrante, révélatrice, selon Micheline Cambron, d'un sentiment de nostalgie. Peu importe que celle-ci se manifeste sous la forme d'un temps circulaire, comme chez Beau' Dommage, ou d'une vectorialité tronquée, comme chez Lysiane Gagnon: il s'agit toujours d'une représentation mythique du temps. Et peu importe que la déchirure du tissu social se manifeste au plan de l'ontogénèse ou de la phylogénèse: il s'agit toujours d'une désillusion.

Plus encore que les conclusions sur lesquelles elle débouche, ce qui fait l'intérêt du modèle proposé par Micheline Cambron, ce sont ses qualités heuristiques. Quel que soit le texte abordé, il est à coup sûr éclairant de s'interroger sur l'organisation du temps et de l'espace, la distribution des rôles et les positions idéologiques qui s'y 
manifestent. Et, uné fois ce modèle mis au point, l'auteur l'applique avec succès à deux œuvres considérées comme typiquement québécoises. Elle démontre ainsi que l'ironie de Deschamps repose sur un équilibre subtil entre les mécanismes d'identification (jeu des pronoms, recours au code linguistique restreint, abondance des lieux communs, absence de perspective historique) et les mécanismes de distanciation (jugements critiques du narrateur, rappels à l'ordre, etc.), qui supposent un autre cadre de référence. C'est également par le décalage entre deux codes - le code de l'écrit et celui de l'oral, le réalisme populaire allié aux conventions tragiques - que Micheline Cambron explique le "scandale" des Belles-Sœurs de Michel Tremblay.

$\mathrm{Si}$, comme le soutient l'auteur, toute œuvre peut se lire comme la mise en forme particulière d'un discours commun, le même type d'analyse doit pouvoir s'appliquer à des œuvres écrites. C'est ce qu'elle s'efforce finalement de démontrer avec les poèmes de Miron et un roman de Ducharme. À propos de l'Homme rapaillé, son analyse l'amène à mettre en lumière l'angoisse d'un sujet aliéné, étranger aux autrès et à lui-même (p. 158), privé de repères spatio-temporels, qui tente à travers le voyage un impossible rapaillage. La démonstration s'achève avec l'Hiver de force de Ducharme qui, selon l'auteur, représente un cas limite, puisque la contestation du discours hégémonique s'y trouve à son tour contestée, sous les traits de la contreculture, par des personnages hors-lieu, hors-temps, hors-norme. Bref, il s'agit d'une mise à distance de la distance, qui se solde par le rejet de toute organisation, éthique, épistémologique ou esthétique.

Contrairement à notre première impression, l'étude de Micheline Cambron s'avère donc d'une extrême cohérence. Elle procède de façon méthodique, reprend les analyses de ses prédécesseurs, les discute, justifie son accord ou son désaccord, sans escamoter aucune difficulté. Au service de sa thèse, elle mobilise avec bonheur les ressources de plusieurs disciplines (sémiologie, sociologie, philosophie, psychanalyse), dont elle manie le vocabulaire spécialisé avec une égale aisance. L'ouvrage fourmille de remarques pénétrantes sur différents sujets. Sa vaste érudition lui permet de citer côte à côte, et toujours à bon escient, Eco, Lacan et Braudel, Bernstein, Kermode et Bakhtine, Robbe-Grillet, Racine et Proust. Le tout, sans la moindre trace de pédantisme ni la moindre dissonance. C'est en effet une des séductions supplémentaires de cet ouvrage que d'allier avec un rare bonheur l'élégance du style à la rigueur de l'argumentation. À lire, pour son instruction et son plaisir.

1 Micheline Cambron, Une société, un récit. Discours culturel au Québec (1967-1976), l'Hexagone, 1989. 